\title{
The Utilization of Needle-Free Acupuncture and Chinese Traditional Medicine Theory to Cure Two Patients Suffering from Different Types of Chronic Urticaria
}

\author{
Qingwen Chen* \\ Wendy Chen Hälsoklinik and Helahuma Akupunktur, Clinical Research Centre, Skånes Universitetssjukhus Malmö, Sweden
}

*Corresponding author: Qingwen Chen, Wendy Chen Hälsoklinik and Helahuma Akupunktur, Clinical Research Centre, Skånes Universitetssjukhus Malmö, Sweden, E-mail: cherqw@hotmail.com

Received date: March 14, 2017; Accepted date: March 20, 2017; Published date: March 28, 2017

Copyright: $\odot 2017$ Chen Q, et al. This is an open-access article distributed under the terms of the Creative Commons Attribution License, which permits unrestricted use, distribution, and reproduction in any medium, provided the original author and source are credited.

\section{Introduction}

People with hypersensitive immune systems are more susceptible to allergens in the environment [1]. This does not usually cause major problems in most people and symptoms will subside after several hours to several days if they are not serious. Sometimes symptoms can last several weeks without any medical treatment. The allergies are normally caused by some special allergens, such as food, pollen, insect stings and even medicine [1]. People with these types of allergies can easily avoid contracting it again by keeping away from allergens. They can also gradually desensitize themselves through sporadic contacts with these allergens or by using allergen immunotherapy [2]. Selfhealing and desensitization show that the human being's body has a system to protect and adapt itself to the environment. But how exactly this system works is still not clear. Problems arise through dysfunctions of this system, such as: an acute allergy changes to be serious or a chronic allergy occurs. People who are suffering with chronic allergies have a low quality of life. Symptoms must be controlled by medicines, or else the allergies will return. In this paper, I will go into detail about a special allergy called chronic idiopathic urticaria, which is very difficult to be controlled by medicines and has no special allergens. This chronic allergy is not considered as a disease by Chinese traditional medicine, but it is in western medicine. According to the Chinese traditional medicine (TCM) theory, chronic allergy is an alarm or symbol to show that the system responsible for protecting and adapting the body to the environment does not work well. This system can be explained by the five elements theory $[3,4]$ (Figure 1), which shows how the body meridians (liver/gall bladder, heart/small intestine, spleen/stomach, lungs/colon, kidneys/bladder) control and influence each other and balance the system. By combining this theory and needle-free acupuncture I treated and cured two patients with different chronic allergy in the span of six months.

\section{Case 1}

A woman, aged 35 year-old, had suffered chronic idiopathic urticaria for 8 months before she started needle-free acupuncture treatment from Wendy Chen Hälsoklinik [5]. In August 2015, the patient suddenly started suffering from urticaria without any reasonable explanation to the origin of the cause. This occurred 11 months after giving birth. There were no reasons for rashes, which were found all over the body and resurfaced every day. These rashes usually appeared in the night and got worse in the morning. Her eyes were swollen and the rashes were extremely itchy. The patient used antihistamine Fexofenadine $4 \times 180 \mathrm{mg}$ every day and histamine- 2 blocker ranitidine $1 \times 150 \mathrm{mg}$ when it was necessary. The patient tried many other things to help her rashes, such as using dietary supplements (vitamins, probiotic bacteria, etc.) in vain. She has also received "Xolair syringes" (Omalizumab) [6] every fourth week since
November 2015 in the hospital. However, new rashes appeared persistently every day with itching and swelling as a result. She often took cortisone to relieve the itching and swelling.

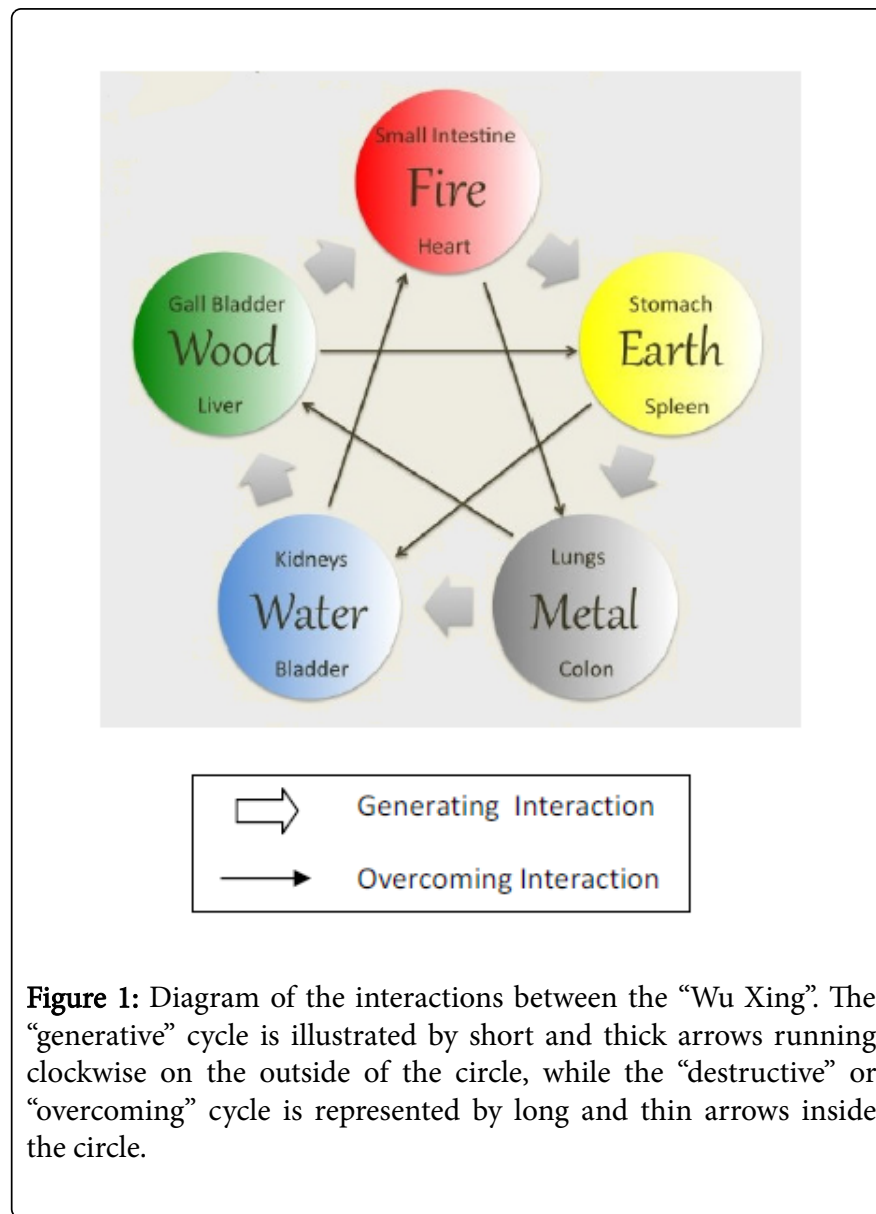

On April 8, 2016, the patient visited Wendy Chen Hälsoklinik. She had taken cortisone in March 2016 and Xolair syringes April 5. The patient was tired and desperate, and had rashes all over the body. After one treatment with needle-free acupuncture, she got more rashes in the evening and had swelling, itching and fever in the morning. Then she used cortisone again. After a one-week treatment with cortisone, the rashes reappeared. During the time from April 14 to April 28 she received four treatments with needle-free acupuncture. The patient felt a significant difference after the treatments. There are almost no rashes after the first two days following the treatment. When they did appear they were not swollen or itchy. After a total of 10 treatments in 5 weeks, the patient felt much better and no longer used cortisone. Her body 
responded well and became stronger. The symptoms reduced, the rashes disappeared quickly and she could sleep well. After a total of 25 treatments in 3 months, the patient took a 3-week vacation abroad. During the holidays she could swim, got tanned and even sweat without getting rashes. She could enjoy life with family again and slept well every day. Rashes would remerge when she had a cold as her defense system was weakened, but they would disappear in three days without cortisone. After 30 treatments in 4 months, she did not get rashes every day and had lowered her antihistamine intake to $2 \times 180$ mg per day. Whenever her immune system was weakened, a small rash would appear but would always subside within a few hours. After 50 treatments in 7 months, the patient was healthy in both body and mind. Her antihistamine intake had been lowered to $1 \times 180 \mathrm{mg}$ per day and according to the TCM the abnormality of her tongue fur has disappeared. She lowered her antihistamine intake to $1 \times 180 \mathrm{mg}$ every other day after 11 months treatment. Nowadays the patient continues to receive treatment once a week.

\section{Case 2}

A woman, aged 48 years old, had suffered chronic idiopathic urticaria for 25 years. The rashes came slowly on her arms and legs, but they were not very itchy. The rashes disappeared naturally in 1-3 weeks without any treatment. When the weather became warm the rashes returned, covered most of her body and were also itchy. Sometimes the rashes would come without any reason. Antihistamines did not help at all and she had tried taking cortisone several times, but the rashes just came back and got worse. During the span of 25 years, the patients tried to use many different physical/natural methods to cure this chronic urticaria, but were unsuccessful. Before the patient began to get the needle-free acupuncture treatment, she did not take any medicine and the rashes always disappeared naturally after 1-3 weeks. The treatment was performed 2-3 times each week. After 6 months the patient had no more rashes reappearing at any time during summer and winter. Nowadays the patient continues to receive treatment once a week.

\section{Treatment}

Each treatment takes 20-30 min: stimulating 12 acupuncture points simultaneously with special low-frequency electric waves from a SSP (silver spike points) therapy machine (NIHON MEDIX CO., LTD. Japan). The SSP therapy machine is utilized in the clinical department of Pain in Japan. Acupuncture points were chosen by using Chinese traditional medicine diagnosis and analysis. They were also adjusted at each treatment according to the previous treatment and also the patient's situation.

\section{Discussion and Conclusion}

Five elements "Wu Xing" $[3,4]$ is the most important theory in TCM. "Wu Xing" explains a process that is fundamental to how the human body and mind are naturally balanced, and that the balance is dependent on the relationship among meridians and organ systems.

The most common symptoms of the chronic urticaria are rashes and itching of the skin, but the rashes that result would usually naturally disappear, which means that the immune system has a self-healing function. According to TCM, chronic urticaria is not considered as a disease, but instead an "alarm signal" for the imbalance among meridians and organ systems. Lungs/metal is one of the organs/ elements in the "Wu Xing" theory. It has a function called "xuanfa" (transpire). For example, many parents are acquaint with the experience that a baby of approximately 6 months of age has a high risk of contracting a viral infection, i.e. high fever for 1-3 days and rashes would appear all over the body after the fever disappears. On the other hand, many people may have experienced skin rashes once or several times in their lives, but recovered naturally by themselves. This is one of the functions of the Lungs/metal, i.e. to transpire the cause of illness out of the body through the skin.

But why were some people unable to easily recover from constantly recurring rashes?

According to the "Wu Xing" theory, when one of five elements is imbalanced, the others elements help create a new equilibrium. For example if an organ such as a spleen/stomach is in imbalance, it may become dysfunctional, so its inhibitory effect to a kidneys/bladder will decrease, then the function of the kidney/bladder will naturally increase. According to the inhibitory relationship among these five elements (Figure 1), eventually it will cause that the function of the spleen/stomach will naturally increase due to the inhibitory effect from a liver/gall bladder to the spleen/stomach decrease. I name this reaction as "buffering effect". When an organ is in imbalance it can be balanced through the "buffering effect". If this effect works well then the body will eventually regain balance. If other organs in the "buffering effect" are not strong enough or are unresponsive to the surrounding changes, then the body is not able to reach a proper new steady equilibrium. The organ imbalance may stay in the body for a long time and have adverse effects on other organs. For example, Long lasting problems in organ liver/gall bladder may cause imbalance in organ lungs/colon or spleen/stomach; Long lasting problems in organ spleen/stomach may also cause imbalance in organ lungs/colon or liver/gall bladder. I name this process as "vicious circle". It is the reason for the occurrence of diseases, such as chronic diseases and cancer. If most of other organs do not aid in the "buffering effect", the imbalance will stay mostly in one of the organs, thus increasing the risk of cancer.

Locating the original or "key" imbalance in these five elements is important for treatment. According to the "buffering effect" and the "vicious circle", to treat other organ imbalances induced by "key" organ imbalance has also effect, which may explain why different TCM doctors or acupuncture therapists are able to treat the same disease with different TCM herbs or different acupuncture points. In Case 1, the "key" organ imbalance is the spleen/stomach, whereas in Case 2, the "key" organ imbalance is the liver/gall bladder. According to the TCM diagnosis and analysis, different acupuncture points were chosen from the beginning of treatment.

It takes a long time to form a "vicious circle" in the body and it always has the tendency to become imbalance. I name this imbalance the "body default state". To break down the "body default state" and build up the equilibrium again, needle-free acupuncture is a fast, stable and non-invasive method for the skin, with minimum administered pain. But it still needs a couple of months and regular treatment. My definition of one period consists of 10 treatments. To cure a patient from chronic urticaria, 5 to 6 periods are required under 6 to 7 months.

In conclusion, the combination of TCM theory and needle-free acupuncture is a new and viable method to cure chronic urticaria. This method may be a viable option to help people suffering from chronic diseases or cancer. 
Citation: Chen Q (2017) The Utilization of Needle-Free Acupuncture and Chinese Traditional Medicine Theory to Cure Two Patients Suffering from Different Types of Chronic Urticaria. J Allergy Ther 8: 249. doi:10.4172/2155-6121.1000249

Page 3 of 3

\section{References}

1. McConnell TH (2007) The Nature of Disease: Pathology for the Health Professions. Lippincott Williams \& Wilkins p: 159.

2. Hoffmann HJ, Valovirta E, Pfaar O, Moingeon P, Schmid JM, et al. (2017) Novel approaches and perspectives in allergen immunotherapy. Allergy.

3. Yu D, Shuanli Z, Peng X, Hai D (2000) Characteristics of the Five Elements of Yin and Yang and New English Translation. Chinese Journal of Integrative Medicine 20: 937.
4. Deng Yu (1999) Fresh Translator of Zang Xiang Fractal five System. Chinese Journal of Integrative Medicine.

5. http://www.wendychen.se.

6. http://www.xolair.com/allergic-asthma/hcp/xolair-for-allergicasthma.html. 